19 Revue d'histoire du XIXe siècle

Société d'histoire de la révolution de 1848 et des

révolutions du XIXe siècle

$32 \mid 2006$

Varia

\title{
De l'écriture à l'événement. Acteurs et histoire de la poésie ouvrière autour de 1840
}

From writing to event. Actors and history of the working-class poetry in the 1840 s

\section{Dinah Ribard}

\section{(2) OpenEdition}

Journals

Édition électronique

URL : http://journals.openedition.org/rh19/1095

DOI : 10.4000/rh19.1095

ISSN : $1777-5329$

Éditeur

La Société de 1848

Édition imprimée

Date de publication : 1 juin 2006

Pagination : 79-91

ISSN : 1265-1354

Référence électronique

Dinah Ribard, « De l'écriture à l'événement. Acteurs et histoire de la poésie ouvrière autour de 1840 », Revue d'histoire du XIXe siècle [En ligne], 32 | 2006, mis en ligne le 30 octobre 2008, consulté le 03 mai 2019. URL : http://journals.openedition.org/rh19/1095 ; DOI : 10.4000/rh19.1095 


\section{DINAH RIBARD}

\section{De l'écriture à l'événement. Acteurs et histoire de la poésie ouvrière autour de 1840}

Paru en septembre 1842 dans la Revue indépendante de Pierre Leroux, le "Second dialogue familier sur la poésie des prolétaires» de George Sand développe la différence entre ce qu'elle appelle «l'avènement des Prolétaires à la poésie» au XIX e siècle, sujet du «Premier dialogue familier», et les quelques cas d'ouvriers et artisans poètes sous l'Ancien Régime ${ }^{1}$. L'opposition est nette : ici la pratique exceptionnelle d'un "métier» qui reste "assimilable en tous points» au "métier manuel» exercé par ailleurs, parce qu'il est inscrit dans des rapports de dépendance et des rapports marchands, là une mission libératrice ${ }^{2}$. La démonstration s'appuie sur un cas, celui d'un poète des années 1630-1650 dont les œuvres viennent alors d'être rééditées (1842), Adam Billaut, dit le «menuisier de Nevers». Tout comme le "Premier dialogue familier» est une bienveillante revue critique des productions récentes d'un certain nombre d'ouvriers-poètes tout autant qu'une réflexion sur le phénomène littéraire qu'ils représentent, le "Second dialogue» est aussi une présentation élogieuse de cette réédition des poèmes de Billaut due à Frédéric Wagnien, un avocat de Nevers. C'est donc l'actualité éditoriale qui détermine le choix d'Adam Billaut pour illustrer la démonstration historique de Sand. D'autres publications, dans ces mêmes années, concourent à cette actualité du poète-menuisier du XVII e siècle pour les producteurs et les premiers lecteurs de la poésie ouvrière du XIX siècle : l'actualité d'un précurseur, l'actualité, donc, d'un jalon de ce qui s'écrit d'emblée comme une histoire ${ }^{3}$.

1. George SAND, "Second dialogue familier sur la poésie des prolétaires», Revue indépendante, septembre 1842, pp. 597-619; voir Frédéric-Gaël THEURIAU, George Sand et les poètes ouvriers, Tours, Éditions FGT, 2003. Sur le phénomène des ouvriers-poètes, voir les travaux de l'équipe LIRE - Littérature, idéologies, représentations (XVIII $-\mathrm{XIX}^{\mathrm{e}}$ siècles), en particulier Hélène MILLOT et al. [dir.], La poésie populaire en France au XIXe siècle. Théories, pratiques et réceptions, Tusson, Éditions Du Lérot, 2005, et ceux de Jacques Rancière, en particulier Alain FAURE et Jacques RANCIÈRE, La Parole ouvrière 1830-1851, Paris, Union Générale d'Éditions, 1976; Jacques RANCIÈRE, La nuit des prolétaires. Archives du rêve ouvrier, Paris, Librairie Arthème Fayard, 1981.

2. "Second dialogue familier...", art. cité, p. 609.

3. Il sera impossible d'étudier ici l'une des pièces de cet ensemble de publications qui actualisent Billaut en en faisant un précurseur de la poésie ouvrière : Le nouvel Adam Billaut, ou Recueil des Poésies de Jean-Charles Jouvenot, ancien artisan serrurier (Baume, Victor Simon, 1831), suivi du nouvel Adam Billaut, ou Seconde partie des productions (Lons-le-Saulnier, Frédéric Gauthier, 1832). Dans ces deux 
Cette rencontre de deux événements, le succès d'une figure d'homme de métier poète dans le deuxième tiers du XVII siècle d'une part, le phénomène (commenté et critiqué comme tel) de l'apparition d'une poésie d'ouvriers sous la Monarchie de Juillet d'autre part, invite à questionner les conditions de l'émergence occasionnelle d'une pratique en réalité ancienne et constante dans le monde du travail. Des artisans et des ouvriers ont régulièrement composé, écrit, produit des textes divers, du Moyen Age à nos jours ${ }^{4}$. Mais il est exceptionnel que cette pratique de la poésie, plus largement de l'écriture, ait fait événement en tant que telle en dehors du monde du travail, et pour d'autres que les artisans et les ouvriers eux-mêmes. Émerge alors la question de ce qui agit en dehors du fait même d'écrire pour produire un tel événement, c'est-à-dire la question des acteurs (ouvriers et autres) impliqués dans cette production et de leurs politiques, qui ne se réduisent pas à une prise de parole. On abordera cette question en partant de l'événement inclus dans l'événement "poètes-ouvriers" que constitue la réapparition d'Adam Billaut au XIX ${ }^{\mathrm{e}}$ siècle. Cette réapparition n'est pas neutre : elle tire avec elle celle d'un monde social où ce qui faisait la carrière d'un auteur, c'est-à-dire lui donnait la possibilité de conquérir un statut, était sa capacité à rendre des services de plume et à se concilier ainsi des protections aristocratiques ou politiques 5 . L'élaboration d'un passé de la poésie ouvrière de la Monarchie de Juillet active ainsi le problème - au-delà même de la conjoncture clientélaire de l'Ancien Régime ${ }^{6}$ - de l'inscription de l'écriture dans des rapports de patronage et de clientèle. On verra dans un deuxième temps que ce problème, fortement énoncé par certains ouvriers-poètes - avant de l'être par les spécialistes du phénomène qu'ils représentaient -, permet de réinterroger cet épisode bien identifié, parce que lié à la question des socialismes et à celle

recueils liés aux activités de l'académie de Besançon, il n'est pas du tout question de Billaut passé le titre. L'auteur se présente comme un artisan ayant dû abandonner le métier du fait d'une paralysie, mais il apparaît plutôt comme un commerçant protégé par les notables locaux et spécialisé dans la production de poèmes d'éloges (de ces notables et de Louis-Philippe). Il s'adresse néanmoins aux ouvriers comme l'un des leurs, et situe quelques-uns de ses poèmes dans des intérieurs ouvriers.

4. Après Natalie Zemon DAVIS (Les Cultures du peuple. Rituels, savoirs et résistances au XVIe siècle, traduit de l'anglais par Marie-Noëlle Bourguet, Paris, Éditions Aubier-Montaigne, 1979) qui donne quelques indications, voir surtout, sur la présence de l'écriture dans le monde des artisans, James S. AMELANG, The Flight of Icarus. Artisan Autobiography in Early Modern Europe, Stanford, Stanford University Press, 1998; sur les conditions locales de la pratique poétique par des travailleurs urbains à la fin du Moyen Age et au début de l'époque moderne, Denis HÜE, La Poésie palinodique à Rouen (14861550), Paris, Librairie Honoré Champion, 2002; sur un cas anglais similaire à celui de Billaut, Bernard CAPP, “John Taylor, 'the Water Poet': A Cultural Amphibian in Seventeenth-Century England”, History of European Ideas II (1989), pp. 537-544; sur l'écriture poétique des ouvriers d'aujourd'hui, voir notamment Marie-Laure LE BAIL, "Ecrire à Rivérac» dans Daniel FABRE [dir.], Écritures ordinaires, Paris, Éditions POL/Centre Georges Pompidou, 1993, pp. 351-372 et Claude F. POLIAK, «Manières profanes de "parler de soi»", Genèses, n 47, 2002, pp. 4-20.

5. Voir Alain VIALA, Naissance de l'écrivain. Sociologie de la littérature à l’âge classique, Paris, Éditions de Minuit, 1985, et, plus spécifiquement sur cette question, Christian JOUHAUD, Les Pouvoirs de la littérature. Histoire d'un paradoxe, Paris, Éditions Gallimard, 2000.

6. Sur cette question, fondamentale en histoire moderne, voir notamment Sharon KETTERING, Patrons, Brokers, and Clients in Seventeenth-Century France, New York/Oxford, Oxford University Press, 1986. 
du rôle des intellectuels, de l'histoire du mouvement ouvrier ${ }^{7}$. En revenant sur le travail des acteurs multiples qui ont constitué l'écriture d'ouvriers en fait d'histoire, il s'agira ainsi d'amorcer une réflexion sur la socialisation de la professionnalisation des écrivains - qui, en ce premier XIX ${ }^{\mathrm{e}}$ siècle, se formule comme sacre de l'écrivain - et, inversement, sur la socialisation de ce qu'on pourrait appeler la désintellectualisation des métiers ouvriers ${ }^{8}$.

\section{LA PRODUCTION D'UN AUTEUR LOCAL}

La publicité donnée par Sand aux Poésies de Maître Adam Billaut, Menuisier de Nevers, pourrait intriguer, s'agissant d'un livre imprimé à Nevers dans un contexte polémique particulier à cette ville et avec le concours de poètes locaux, Antony Duvivier (auteur en 1840 d'un recueil intitulé Une voix du Morvan) et François Rouget, qui se font écho dans l'éloge d'une gloire nivernaise ${ }^{9}$. En réalité, on touche là un des traits caractéristiques de l'événement "poètes-ouvriers» des années 1840. Si la poésie composée par des ouvriers fait alors événement, c'est-à-dire est constituée comme événement, c'est bien en effet comme révélation et irruption sur la scène publique d'écritures issues d'une expérience du confinement social, autrement dit de l'assignation à des lieux très spécifiques ${ }^{10}$. Dans bon nombre de cas, cette fixation

7. Sur la question de l'événement et de l'utopie, voir Michèle RIOT-SARCEY, Le Réel de l'utopie. Essai sur le politique au XIXe siècle, Paris, Éditions Albin Michel, 1998.

8. Sur ce dernier point, voir William H. SEWELL, Gens de métiers et révolutions. Le langage du travail, de l'Ancien régime à 1848, traduit de l'anglais par Jean-Michel DENIS, Paris, Éditions AubierMontaigne, 1983. Le présent article s'inscrit dans la préparation d'un livre sur le travail intellectuel et l'écriture des artisans et des ouvriers à partir du cas d'Adam Billaut et de ses historiographies, entre XVII e et XXe siècles.

9. «Second dialogue familier...», art. cité, pp. 597-598: «On nous apporta dernièrement une nouvelle et magnifique édition des Poésies de maître Adam Billaut, que M. Ferdinand Wagnien, avocat, vient de collationner avec soin, et d'offrir au public comme un monument élevé à la gloire de son compatriote [...] M. A. et M. Z. [...] reprirent à ce propos leur ancienne discussion sur l'avènement des Prolétaires à la poésie, en commençant par admirer ensemble ce beau volume, imprimé à Nevers même avec élégance, recomplété par les soins vigilants de sympathiques admirateurs, rendu à sa véritable orthographe ancienne [...] et enrichi des portraits intéressants et authentiques de maitre Adam, du grand Condé, de Christine de Suède, des princesses de Gonzague, etc. On y a joint une vue du vaste château des ducs de Nevers, où le poète artisan porta si souvent ses stances et ses sonnets [...] et enfin une vue de la maisonnette plus que modeste où le vieux Adam acheva tranquillement ses jours dans une philosophique pauvreté [...] Une Notice fort bien faite par M. Ferdinand Denis, ouvre le volume; et une jolie Epître en vers adressée à la mémoire de maître Adam par Rouget, le tailleur poète de Nevers, le termine, et en complète l'illustration.»

10. Voir par exemple l'avant-propos des Poésies sociales des ouvriers, le recueil publié par Olinde Rodrigues en 1841, aussitôt accueilli, pour le saluer ou pour le critiquer, comme un événement : «j’ai hâte de le proclamer [...] : j'ai trouvé dans les ateliers, sous le toit des mansardes, nombre d'hommes de cœur et d'intelligence, qui déjà, dans le cercle de leurs habitudes, de leurs relations, s'occupent activement et avec un succès croissant à propager par leurs écrits, ou par leurs chants, des idées d'ordre et de paix, basés sur une foi ardente en un meilleur avenir" (Poésies sociales des ouvriers, réunies et publiées par Olinde RODRIGUES, Paris, Paulin, 1841, p. II). Voir aussi la préface donnée par Eugène Sue au recueil de Savinien Lapointe (Une voix d'en bas. Poésies par Savinien Lapointe, ouvrier cordonnier, Paris, Adolphe Blondeau, 1844), tout entière centrée sur la question du lieu d'où doit parler qui entend parler du peuple. 
sociale est aussi géographique : les poètes ouvriers apparaissent comme des poètes locaux, liés à une ville ou à une province ${ }^{11}$. La coopération d'acteurs de la scène nationale et de la scène locale - lesquels se définissent par là même comme aptes à circuler et à intéresser ailleurs, tandis que les premiers y gagnent une mise en avant de leur capacité d'intervention - dans la production d'une poésie de l'enracinement se trouve par là favorisée, parce qu'appelée à être donnée à voir dans les publications qui opèrent cette production et sa mise en récit.

Les Poésies de Maître Adam manifestent une de ces coopérations. Ce bel in-quarto, qui fait l'objet d'annonces publicitaires dans les différents journaux nivernais et se présente comme l'édition définitive de Billaut, savamment annotée par le fils d'un personnage de la vie politique régionale ${ }^{12}$, est orné d'illustrations dues notamment au peintre romantique parisien Achille Devéria, et ouvert par une "notice biographique et littéraire» rédigée par Ferdinand Denis, conservateur de la Bibliothèque Sainte-Geneviève. En relations avec Sainte-Beuve, Sand ${ }^{13}$, Ballanche, Denis est l'auteur d'écrits divers sur les auteurs lusophones et les sciences occultes, mais aussi sur l'histoire médiévale et les traditions populaires dont il fait figure de spécialiste. Un épisode de son journal, contemporain de la parution des poésies de Billaut, éclaire sa participation à l'entreprise : il raconte avoir été invité par Augustin Thierry à assister à une lecture par leur auteur des poésies de Jasmin, coiffeur agenais (présent à ce titre dans maintes publications sur les poètes-ouvriers) et poète

11. L'avant-propos d'un autre recueil de poésie ouvrière, celui de François Gimet, identifie de manière explicite position sociale et origine régionale : «Notre plume et nos goûts se sont identifiés avec les coutumes locales des ouvriers-poètes; nous les avons rassemblés dans un même tableau [...] en conservant à chacun [...] sa fraîcheur et sa grâce, quelque agreste et quelque sauvage qu'elles soient" (Galerie d'ouvriers-poètes. Les Muses prolétaires. Adam Billaut, Jean Reboul, Jasmin, Magu, Marius Fortoul, Rouget, Louis Voitelain, Charles Poncy, Auguste Abadie, Reine Garde, Paris, Émile Fareu, 1856, pp. 5-6), et plus loin, pp. 12-13 : «Par un goût qui nous est particulier, nous avons suivi chaque ouvrier-poète dans son atelier, dans son chantier [...] Nous avons parlé leur jargon, leur patois, nous les avons surpris avec leurs amis, avec leur famille et nous les avons suivis dans toutes les phases de leur vie». Lorsque Lamartine écrit sur Reboul, poète nîmois, il le compare à un laurier poussant sur le Pont du Gard ou les Arènes : la poésie de ce boulanger catholique est pour lui une manifestation du génie propre des lieux.

12. Le père de Wagnien, ancien président du tribunal de commerce et receveur municipal de Nevers, est un républicain très actif dans les années 1840. Lui-même avocat, conseiller municipal, lié aux fouriéristes, Frédéric Wagnien publie différentes brochures et devient en 1842 l'un des rédacteurs de la Revue de la Nièvre, journal d'opposition modéré rattaché, selon ses propres termes, "à la gauche dynastique» (Coup d'œil sur le Nivernais, par M. Frédéric Wagnien, avocat, Nevers, C. Sionest, 1846, p. 27), mais il écrit aussi dans L'Association plus radicale de Claude Tillier. Sur ces deux figures, voir Victor GUENEAU, Dictionnaire biographique des personnes nées en Nivernais ou revendiquées par le Nivernais, qui, par leurs travaux, leurs services, leurs mérites, leurs vertus ou leurs crimes, ont mérité de n'être pas oubliées, Nevers, Mazeron frères, 1899; et André LEGUAI et Jean-Bernard CHARRIER [dir.], Histoire du Nivernais, Dijon, Éditions universitaires de Dijon, 1999, pp. 305 et $s q$.

13. On peut remarquer que dans le premier article de la Revue indépendante qu'elle consacre aux poètes populaires sous le pseudonyme de Gustave Bonnin ("Sur les poètes populaires", novembre 1841 : il s'agit du premier numéro de la revue), Sand développe le même raisonnement que dans le "Second dialogue familier [...]» sur la succession historique des poètes issus de la noblesse (jusqu'au XVII siècle), puis de la bourgeoisie (XVII ${ }^{-}$-XVIII ${ }^{e}$ siècles), enfin du peuple, mais sans parler d'Adam Billaut. Le retour sur son cas signale le fonctionnement en réseau des acteurs de l'événement "poètes-ouvriers". 
de langue d'oc ${ }^{14}$. Le biographe d'Adam Billaut, qui ne manque d'ailleurs pas de comparer celui-ci à Jasmin, comme s'il y avait continuité de l'un à l'autre, n'est pas déplacé dans ce monde d'acteurs parisiens de l'institutionnalisation d'une histoire locale considérée comme constitutive de l'histoire nationale et de promoteurs érudits d'une mise en valeur des héritages culturels différenciés - y compris dans leur dimension linguistique - qui nourrissent selon eux l'identité française ${ }^{15}$. Et de fait, la réédition des poèmes du menuisier de Nevers est aussi une pièce parmi d'autres, innombrables, de l'immense mouvement de célébration écrite de la localité au XIX siècle, ce que Daniel Fabre appelle la « révolution, énorme mais silencieuse, des contenus et des ancrages sociaux et territoriaux de l'écrit" ${ }^{16}$.

La mise en scène de la coproduction du poète local par des célébrités parisiennes et des acteurs nivernais avait d'ailleurs commencé dès avant la parution des Poésies de Maître Adam. Cette parution avait en effet été préparée par la publication dans l'Écho de la Nièvre, en décembre 1835 et janvier 1836, de six pièces de Billaut présentées comme inédites. S'ensuivit une polémique mettant aux prises dans les colonnes du journal d'une part un professeur au collège de Nevers, Morellet ${ }^{17}$, qui entendait démontrer que ces poèmes n'étaient pas inédits et manifesta rapidement son projet de procurer une édition des œuvres de Billaut concurrente de celle annoncée par Wagnien, et d'autre part celui-ci, Norbert Duclos (le rédacteur de l'Écho de la Nièvre), et l'homme qui avait envoyé à Wagnien les pièces en cause, à savoir Abel Hugo, qui répondit violemment à Morellet. Dans la même période (6 mars 1836), Abel Hugo publiait dans L'Écho un article en forme de lettre à son frère

14. Ferdinand-Jean DENIS, Journal 1829-1848, publié par P. Moreau, Fribourg, Hess frères et Paris, Plon, 1932, pp. 113-115. Sur Jasmin, voir Emmanuel LE ROY-LADURIE, La Sorcière de Jasmin, Paris, Éditions du Seuil, 1983 et Jasmin. Actes du colloque d'Agen (9-11 octobre 1998), textes réunis par Claire TORREILLES et François PIC, Toulouse, Centre d'étude de la littérature occitane et Bordeaux, William Blake et Co, 2002.

15. Sur ce mouvement et ses acteurs, notamment Augustin Thierry, voir Stéphane GERSON, The Pride of Place. Local Memories and Political Culture in Nineteenth-Century France, Ithaca et Londres, Cornell University Press, 2003. Gerson mentionne l'intérêt du cercle intellectuel de Thierry pour les patois au début des années 1840, p. 214.

16. "L'histoire a changé de lieux" dans Alban BENSA et Daniel FABRE [dir.], Une histoire à soi. Figurations du passé et localités, Paris, Éditions de la Maison des sciences de l'homme; 2001, pp. 1341, citation p. 21. Daniel Fabre signale ibidem que "quelle que soit l'unité d'espace que l'on retienne - commune, département ou province - la France et ses voisins européens ont connu au XIX ${ }^{\mathrm{e}}$ siècle une mutation considérable des référents de l'écrit et de l'imprimé : désormais la localité écrase quantitativement et les écrits religieux dont la domination ancienne fut durable et les nouvelles productions de la littérature de masse. " Sur le cas de la Nièvre, voir Guy THUILLIER, "Les historiens locaux en Nivernais de 1815 à 1840 ", Actes du 101 Congrès national des sociétés savantes, Lille, 1976, Paris, Bibliothèque Nationale, 1978, tome 2, pp. 349-364.

17. Sur ce personnage, neveu de l'académicien du XVIII e siècle, et qui s'occupa lui aussi d'érudition locale dès sa nomination à Nevers, comme le faisaient beaucoup d'enseignants - correspondant du Comité des travaux historiques depuis 1838, il avait participé, avec Antony Duvivier et un autre personnage local, Barat, à l'Album du Nivernais, publié de 1838 à 1842 -, voir André THUILLIER, "Une carrière universitaire au XIX" siècle. J. N. Morellet (1806-1890)", Mémoires de la société académique du Nivernais, 1971, pp. 84-90. Sur la polémique à propos d'Adam Billaut, voir la notice de la «Physiologie du professeur de rhétorique» dans Claude TILLIER, Pamphlets (1840-1844), éditions critique par M. Gérin, Paris, A. Butaut et Nevers, Mazeron frères, 1906, pp. 173-177. 
Victor, consacré à une description archéologique de la cathédrale de Nevers et bientôt reproduit dans sa France historique et monumentale ${ }^{18}$. D'emblée, la production des expressions artistiques du peuple français à Nevers apparaissait donc comme l'affaire d'un personnel nivernais capable de mobiliser des acteurs de la scène parisienne - comme une affaire tout à la fois locale et délocalisée.

Cette politique collective de la poésie locale a des enjeux politiques. $L^{\prime} E ́ c h o$ de la Nièvre, c'est-à-dire le journal gouvernemental ouvertement protégé par la préfecture, soutenait Wagnien contre Morellet, mais L'Association, le journal républicain radical dirigé par Claude Tillier, allait prendre le relais ${ }^{19}$. Le 24 février 1842, c'est-à-dire dans le numéro où était annoncée pour la première fois la parution des Poésies de Maître Adam, paraissait aussi la «Physiologie du professeur de rhétorique», un pamphlet de Tillier dirigé de manière transparente contre Morellet ${ }^{20}$. Les deux camps se réunissaient donc dans une polémique littéraire autour d'une figure politiquement neutralisable (un ouvrier-poète qui est aussi un héros du passé régional), menée par un personnel sinon de notables, du moins de "capacités" qui forgeaient là un peu de leur aptitude à prendre part au gouvernement - c'est-à-dire au gouvernement local ${ }^{21}$. Il est sans doute significatif, de ce point de vue, que l' "Épître à Maître Adam» du tailleur-poète François Rouget, insérée dans les Poésies de Billaut "pour être agréable [aux] lecteurs, surtout aux Nivernais", soit amputée de toute une partie consacrée, selon Wagnien, à «une longue dissertation sur la littérature actuelle [...] étrangère au sujet principal» ${ }^{22}$. L'ouvrier-poète, pendant contemporain de l'auteur qu'il célèbre, est ainsi confiné à son appar-

18. France historique et monumentale. Histoire générale de France depuis les temps les plus reculés jusqu’à nos jours, illustrée et expliquée par les monuments de toutes les époques [...], par A. Hugo, auteur de l'histoire de Napoléon et de la France pittoresque, Paris, Delloye, 1836-1853. La France pittoresque, publiée en 1835 chez le même éditeur, est illustrée de gravures dues à Devéria. Les numéros de l'Écho de la Nièvre. Journal politique, littéraire et industriel où apparaît cette polémique manquent à la Bibliothèque nationale de France; ils peuvent être consultés à la Bibliothèque municipale de Nevers, qui ne possède toutefois pas la collection complète de ce périodique.

19. Sur ces journaux, voir Guy THUILLIER, "La presse nivernaise au XIX e siècle», extrait des Annales de Bourgogne, tome 38, 1966.

20. Le 27 février 1842, la rubrique théâtrale de L'Association annonce également la représentation d'un vaudeville sur Adam Billaut au théâtre de Nevers (Les Chevilles de Maître Adam, menuisier de Nevers ou les Poètes artisans, par Fancis et Moreau), avec ce commentaire, p. 3 : "Aucune pièce ne saurait offrir plus d'attraits que les productions auxquelles s'attache un intérêt local [...]. Le menuisier de Nevers est le seul poète populaire du siècle de Louis XIV. Sa gloire et sa popularité n'ont point pâli [...] Nous serons donc enchantés de voir reparaître au milieu de nous, sur notre petit théâtre qui occupe une partie du château des anciens ducs. C'est là que le poète venait dire ses vers à la séduisante Marie de Gonzague; c'est là que les jeunes princesses récitaient elles-mêmes ceux que maître Adam composait [...] Les Nivernais, nous en sommes sûrs, ne laisseront pas échapper cette occasion de prouver par leur empressement que leur vieux poète excite encore aujourd'hui les plus vives sympathies ".

21. Voir Philippe RÉGNIER, «L'institution et son en-dehors. La critique littéraire des saint-simoniens » dans Michel ESPAGNE et Michael WERNER [dir.], Philologiques I. Contribution à une histoire des disciplines littéraires en France et en Allemagne au XIXe siècle, Paris, Éditions de la Maison des sciences de l'homme, 1990, p. 211-237, pour une analyse du même phénomène chez des acteurs bien plus centraux, les saint-simoniens, ex-saint-simoniens et relations des saint-simoniens dans le monde politique, la presse et les institutions culturelles parisiennes. p. 605 .

22. Poésies de Maître Adam, Menuisier de Nevers, Nevers, J. Pinet, 1842, respectivement p. 599 et 
tenance locale dans le livre même où les "capacités " nivernaises le projettent sur une autre scène. Or le passage disparu montre précisément que Rouget avait entrepris de se constituer lui-même, par son écriture, en acteur parfaitement apte à intervenir sur cette autre scène. À partir du cas de Jean Reboul, boulanger-poète nîmois protégé par les légitimistes locaux et rendu célèbre par Lamartine et Alexandre Dumas, il y dénonce en effet les alliances profitables conclues entre des auteurs parisiens désireux d'apparaître comme des découvreurs et des ouvriers-poètes qui achètent l'influence de ces célébrités au prix d'un renoncement à la vérité de leurs pratiques d'écriture, et au sens politique qui devrait être le leur ${ }^{23}$.

\section{LA PRODUCTION DE LA LITTÉRATURE}

Rouget n'est pas un cas unique. Jacques Rancière signale, à propos de Gabriel Gauny, le philosophe et poète central de La Nuit des prolétaires, que la signification sociale de cet échange entre auteurs professionnels établis et poètes-ouvriers (protection contre renforcement de cet établissement par le consentement au patronage) était clairement perçue par certains de ceux-ci. Gauny commente en effet le conseil que lui donnait Victor Hugo au moment où il débutait en poésie, "Soyez toujours ce que vous êtes», en rétablissant la subordonnée implicite : "afin que nous puissions demeurer ce que nous sommes» ${ }^{24}$. La "représentation poétique» du peuple proposée par Eugène Sue, à propos de Savinien Lapointe, comme substitut à sa "représentation politique" absente, implique de fait que le poète représentant reste là où il est, quitte à être aidé à se faire entendre par des écrivains reconnus à la fibre

23. Poésies de François Rouget, tailleur à Nevers, Paris, Arnauld de Wresse, 1857, "Epître à Maître Adam", p. 20-24 : "À Nîmes, un boulanger, des beaux-arts amateur, / Et de vers assez bons assez modeste auteur / Poursuivait dès longtemps sa docte fantaisie, / Et faisait de la pâte et de la poésie. / [...] Un travail manuel n'exclut pas la science. / [...] Jean Reboul, - c'est le nom du poète-ouvrier, / Cultivait donc les arts ensemble et son métier, / [...] Un jour, à son logis un noble voyageur / Se présente et s'annonce en qualité d'auteur. / C'était le grand Dumas, alors roi du théâtre, [...] / Jean Reboul, étonné, le salue humblement. / [...] L'étranger lui répond : [...] / Un frère en Apollon vient vous baiser les mains. / [...] Vous serez imprimé sur beau papier vélin, / Ou j’y perdrai plutôt mon grec et mon latin. / - Monsieur, reprit Reboul, votre bonté s'abuse, / Et vous portez trop haut une modeste muse; / Je sais juger mes vers, et jamais je ne lis / Racine et Despréaux, mes auteurs favoris, / Sans renoncer huit jours à toute poésie. / [...] - Trop modeste, mon cher; d'ailleurs ne parlez point / De ces auteurs. Soyons bien d'accord sur ce point : / Vous êtes boulanger, sans art et sans lecture, / Vous ne connaissez rien de la littérature; / Vous n'avez rien appris de cent auteurs divers, / Et c'est par pur instinct que vous faites des vers. / [...] De vous répandre, ami, laissez-moi tout le soin; / [...] Pour vos œuvres d'abord, je fais une préface, / Et puis un mien ami, poète député, / Dont le nom glorieux est justement vanté, / [...] Une lettre par lui nous sera délivrée; / Sa lettre et ma préface enflant votre recueil, / Je vous promets partout un favorable accueil. / À des soins si pressants, qui pourrait se soustraire? / - J'y consens, dit Reboul, faites-en votre affaire. / Et depuis, ses beaux vers, par le ciel inspirés, / Réimprimés trois fois, sont toujours admirés. / Mais que j’aimerais mieux le rimeur prolétaire, / Servant dans ses écrits la cause populaire, / Et consacrant son luth, si justement vanté, / À vous, sainte patrie et sainte liberté!» Le recueil de Rouget contient de nombreux poèmes consacrés à la figure d'Adam Billaut.

24. Jacques RANCIÈRE, "Le Prolétaire et son double, ou le Philosophe inconnu ", dans Les Scènes du peuple, Lyon, Horlieu éditeur, 2003, pp. 21-33, citations p. 25. 
populaire elle aussi reconnue, comme ceux dont les lettres à Lapointe forment le péritexte d'Une voix d'en bas, Léon Gozlan, Victor Hugo et Béranger ${ }^{25}$.

Non seulement donc très peu d'ouvriers poètes, passée la conjoncture des années 1840 , feront une carrière d'auteur, voire continueront à être publiés, mais la publication même des "poètes-ouvriers", c'est-à-dire un faisceau d'opérations où agissent ensemble des praticiens de l'écriture séparés par leurs appartenances de classe, contribue en réalité à renforcer la différence entre leurs positions. Cette analyse est contemporaine de l'événement et constitutive de son immédiate mise en histoire - il en existe des versions roses aussi bien que des versions noires. On peut remarquer aussi qu'elle construit un face-à-face où l'auteur professionnel établi, le grand écrivain, vient en quelque sorte remplacer l'aristocrate d'Ancien Régime dans le rôle de patron - ce qui pourrait aller jusqu'à neutraliser la question de la classe. L'analyse doit donc elle-même être analysée. Or elle peut l'être, et elle peut aussi être enrichie, si l'on prend justement en compte ce qu'elle omet : la mobilisation des réseaux de notables locaux dont on a parlé, et qui ne sont mentionnés ni par Rouget ${ }^{26}$ - alors qu'il dénonce dans plusieurs poèmes les écrivains bourgeois, notamment l'indifférence d'Hugo, de Dumas et de Scribe à la misère d'Héségippe Moreau - ni par Gauny, mais aussi l'action de certains des ouvriers investis dans l'événement, et qui n'y interviennent pas seulement par le fait même d'écrire et de devenir poètes.

L'une des formes de cette action, la plus évidente avec la fondation des journaux ouvriers, se donne à lire dans beaucoup de recueils de poésie ouvrière. Leurs auteurs ne cessent pas de mentionner élogieusement les œuvres des autres écrivains ouvriers, de montrer qu'ils les ont lus, de les défendre contre leurs détracteurs et de leur adresser des pièces, bref de mettre en scène un groupe solidaire et cohérent. Cette solidarité, d'autre part, se manifeste par la circulation et le réemploi de formules susceptibles de donner des titres à la reconnaissance d'un livre à un autre. Lorsque Louis Pélabon, ouvrier à l'Arsenal de Toulon, publie son Chant de l'ouvrier (1842), par exemple, il ne manque pas de se comparer à Adam Billaut : «Après de pareilles productions Pélabon serait en quelque sorte en comparaison avec Maitre Adam menuisier de Nevers, qui est sans contredit l'un des hommes les plus extraordinaires de son siècle. On a peine à concevoir qu'un artisan privé de toute instruction ait pu rimer les vers que nous avons de lui. Beaucoup de gens ne connaissent de Maître Adam que sa chanson : Aussitôt que la

25. Une voix d'en bas, ouv. cité., préface, p. xv : «Nous le répétons, rien ne nous paraît donc plus touchant, plus beau que de voir des hommes d'une intelligence, d'un talent aussi élevé que M. Savinien Lapointe, rester ouvriers comme leurs frères, vivre de leur vie de rude labeur, afin d'être toujours l'écho de leurs douleurs, de leurs vœux, de leurs espérances, et, à défaut de représentation politique, créer ainsi une sorte de représentation poétique, à laquelle la puissance de leur voix donne autant de retentissement que d'importance."

26. Reboul, on l'a dit, était bien protégé par les notables catholiques et légitimistes de Nîmes, et devint même représentant du peuple pour leur compte après 1848. Mais ce n'est pas de cela que Rouget parle : il n'évoque que le patronage de Lamartine et Dumas. 
lumière, et son fameux rondeau commençant et finissant par ces mots : pour te guérir, mais on trouve encore dans les chevilles ${ }^{27}$ de notre menuisier, parmi quelques négligences, une foule de vers charmants. On peut citer comme un modèle de poésie et de philosophie, les stances à un de ses amis qui le sollicitait d'aller à la cour. [...] Tous les poètes du temps lui adressèrent des vers, et le grand Corneille ne dédaigna pas d'y joindre son hommage ${ }^{28}$.

Le passage révèle une grande réactivité de Pélabon à l'actualité des publications sur la poésie ouvrière : la réédition de Billaut et le dialogue de Sand sont exactement contemporains de son livre. Mais cette réactivité s'inscrit dans ce que le recueil affiche, notamment par des pièces croisées : la proximité avec d'autres poètes ouvriers, notamment un Toulonnais plus célèbre, correspondant de Sand (qui préfacera son Chantier en 1844) et auteur publié dans la Revue indépendante, Charles Poncy.

Dans la correspondance entre Agricol Perdiguier - lui-même compositeur et éditeur de chansons liées au monde du compagnonnage, et présent à ce titre dans les Poésies sociales d'Olinde Rodrigues - et Sand, deux lettres du premier évoquent les articles de la seconde sur les poètes-ouvriers. L'une (21 mars 1842) mêle à parts quasi égales une demande d'intervention en faveur des beaux-parents de Perdiguier et une suggestion : "Je serais bien content, si après avoir fait un second article sur Magu ${ }^{29}$, vous pourriez vous occuper des autres artisans poètes, et surtout de Lebreton et de Roly. Dans ce moment, la presse quotidienne semble dédaigner les productions littéraires des artisans [...] elle redoute la concurrence de ceux qu' elle a trop longtemps indignement trompés. Aussi elle se garderait bien de leur dire : vous faites bien, continuez. Vous comprenez que quand je parle de la grande presse [...]; je parle de la bourgeoisie, puisque les écrivains bourgeois [...] cherchent à décourager les prolétaires les plus intelligents et les plus dévoués; c'est à vous, Madame, qui souhaitez sincèrement le bien [...] à détruire leur tactique, à encourager les artisans poètes et prosateurs, à leur donner confiance en euxmêmes, afin qu'ils puissent marcher en avant avec toujours plus d'assurance et se faire suivre par un nombre toujours plus grand, dussent, comme vous l'avez dit vous-même, quelques suicides de plus avoir lieu chaque année [...] Au reste, si la publication d'un livre utile a causé la mort de mon ami Boyer, mort qui m’a profondément affligé [...], l'ignorance et l'abrutissement causent tous les jours mille morts dont on ne parle pas.» ${ }^{30}$

27. Les Chevilles est le titre du seul recueil publié de son vivant par Billaut (Les Chevilles de Maître Adam, Menuisier de Nevers, Paris, Toussait Quinet, 1644).

28. Le Chant de l'ouvrier. Poésies diverses par Louis Pélabon, Draguignan, P. Garcin, 1842, Préface, pp. 10-11; Pélabon parle de lui-même à la troisième personne. Sur cet ouvrier qui écrit aussi en provençal et l'autre poète toulonnais, Charles Poncy, voir Maurice AGULHON, Une ville ouvrière au temps du socialisme utopique. Toulon de 1815 à 1851, Paris/La Haye, Mouton, 1970, notamment pp. 54-55, pp. 71-78, pp. 155-156.

29. Sand préfacera ses Poésies en 1845 , et sa préface paraîtra aussi dans La Presse.

30. Agricol PERDIGUIER, Correspondance inédite avec George Sand et ses amis, lettres choisies et commentées par Jean Briquet, Paris, Éditions Klincksieck, 1966, pp. 63-64. L'histoire de Boyer, mort 
Le 23 juillet 1843, Perdiguier raconte d'abord à Sand l'histoire d'un menuisier-poète de Fontainebleau, Alexis Durand, privé de la pension qu'il recevait pour des vers irrévérencieux reproduits dans la lettre. Avant de déclarer qu'il a refusé de faire pour Flora Tristan ce qu'il fait pour sa correspondante - c'est-à-dire de signaler sa loyauté envers cette dernière - et d'envoyer «bien des choses aimables de ma part à MM. Chopin, votre fils et votre frère», il réitère sa suggestion de 1842 : "Vous avez parlé dans le temps, Madame, des vers de Magu, des vers de Beuzeville, mais vous n'avez encore rien dit de ceux de Durand, de Lebreton, de Roly, de Vinçard, de Poncy, etc.... Si vous donniez l'analyse de tous les ouvrages en prose et en vers publiés par des ouvriers, depuis maître Adam jusqu’à nos jours, je suis assuré que vous feriez un livre populaire et très utile.

Les ouvriers commencent à vous connaître et à vous aimer; depuis quelque temps je suis presque commissionnaire en librairie, je place de nombreux exemplaires du Compagnon du Tour de France, mais à peine a-t-on lu cette première partie qu'on s'empresse de demander la continuation [...] Je pense que vous serez assez bonne pour satisfaire tant d'impatience, tant de lecteurs artisans qui vous prient de penser à eux» ${ }^{31}$.

Obligé de Sand, dont il s'efforce - et surtout dit s'efforcer - de faire la publicité en milieu ouvrier, Perdiguier agit aussi ici en patron des autres poètes-ouvriers auprès d'elle. Il met certains noms en avant, défend une figure possiblement équivoque comme celle de Durand, d'abord protégé de LouisPhilippe, incite Sand à encourager les auteurs ouvriers par l'écriture - ce qui peut renvoyer à la rédaction d'articles de presse, mais aussi à l'activité de conseillère éditoriale que Sand exerce également, voire à la correspondance, qui permet au poète ainsi distingué de se prévaloir de la protection de l'écrivain -, fournit même des arguments et l'idée du plan d'un ouvrage.

Les ouvriers-poètes - en particulier, mais pas seulement, ceux d'entre eux qui accèdent à des formes de notabilité qui se traduiront parfois par une carrière politique - agissent donc eux-mêmes comme des auteurs professionnels. Ils construisent des réputations par des éloges (et des dénonciations) croisés, entrent dans des réseaux d'interconnaissance et d'échanges de services qu'ils contribuent par là même à étendre, produisent l'actualité dans laquelle euxmêmes et d'autres peuvent s'insérer ${ }^{32}$. De là, aussi, leur identification à la

de désespoir de ne pas voir ses œuvres reconnues, a servi d'argument aux détracteurs des poètes-ouvriers, dont le plus célèbre et le plus réfuté (notamment par Sand et Savinien Lapointe) est Lerminier, auteur d'un article cinglant dans la Revue des Deux Mondes ("De la littérature des ouvriers», 15 décembre 1841).

31. Correspondance inédite..., ouv. cité, pp. 67-69. Sand répond qu'elle travaille à un autre article sur les ouvriers-poètes et à une suite du Compagnon du tour de France, le roman qu'elle a écrit sur l'expérience compagnonnique qu'elle connaît par Perdiguier.

32. Un signe a contrario de ce travail est repérable dans les deux versions successives de la préface du dialogue sur Adam Billaut écrit par Perdiguier lui-même en 1843, mais publié en 1850 puis en 1863. La première version, très politique, contient aussi une liste des noms de poètes-ouvriers qu il voulait voir célébrer par Sand : "J'avais eu la pensée, en l'année 1843, de faire un travail complet sur les ouvriers qui ont écrit soit en vers, soit en prose. J'avais, à cet effet, réuni de nombreux documents; mais, par des 
conception même de la littérature qui ne peut que creuser l'écart entre les écrivains bourgeois établis et les ouvriers devenus écrivains. Cette conception développée par Sand, mais aussi par Perdiguier dans son dialogue sur Adam Billaut, aussi bien que dans leur correspondance, repose en effet sur l'idée d'un nécessaire désintéressement (ou plutôt d'un nécessaire devenirdésintéressé, pour ainsi dire) de la pratique littéraire. Selon eux, celle-ci doit toujours plus se distinguer du "métier» qu'elle était encore au XVII siècle, c'est-à-dire pour Corneille comme pour Billaut, que Perdiguier et Sand rapprochent, pour devenir une activité missionnaire. De cette vérité à venir de la littérature, les poètes-ouvriers sont pour ceux qui parlent d'eux les preuves et les témoins par cela même qu'ils acceptent - c'est-à-dire écrivent qu'ils acceptent - de ne pas faire d'elle leur métier, de ne pas faire carrière littéraire.

La figure extrême de ce piège discursif qui peut être aussi un moyen d'action pour ceux qui s'en saisissent pour eux-mêmes, surgit au détour d'un passage de l'autobiographie de Sand. Évoquant la «mendicité organisée» autour des "artistes» - c'est-à-dire des artistes aisés et établis - à laquelle elle a été confrontée à Paris parce qu'elle se refusait à fermer sa porte à la misère, elle signale l'usage des histoires dramatiques de certains ouvriers-poètes par ceux qui venaient la supplier : «Le sort d'Elisa Mercœur et d'Hégésippe Moreau sert désormais de thème et de menace", écrit-elle, "à tous les poètes qui ne rougissent pas de mendier, et qui se disent trop grands hommes, pour faire un autre état que de rêver aux étoiles" ${ }^{33}$. Ce récit méprisant pointe, sous une forme caricaturale, la double nature de «l'avènement des Prolétaires à la

circonstances indépendantes de ma volonté, mon projet ne put être réalisé [...] Les écrivains-ouvriers dont j'aurai à parler, s'il est décidé qu'il est bon de poursuivre un travail de cette sorte, seront, parmi les plus anciens : Olivier BASSELIN, le fouleur; maître ANDRE, le perruquier [...]. Viendront ensuite le boulanger REBOUL; le perruquier JASMIN; [...] les imprimeurs Hégésippe MOREAU, LACHAMBAUDIE, VOITELIN; le tisserand MAGU; le potier d'étain BEUZEVILLE; l'imprimeur sur indiennes LEBRETON, le cordonnier LAPOINTE; le fabricant de mesures linéaires VINÇARD; le maçon PONCY [...]", Maître Adam, menuisier de Nevers, Dialogue, par Agricol PERDIGUIER, menuisier, représentant du peuple, Paris, Marcel, 1850, pp. 5-6. La deuxième version ne mentionne plus aucun contemporain : "Les ouvriers écrivains datent de loin; Moïse, David, Elisée, Amos, Confucius, Socrate [...] travaillèrent des mains [...] Jean-Jacques Rousseau fut apprenti graveur et la pauvreté ne le quitta jamais [...] Mais soyons moins ambitieux; ne parlons que de ceux qui naquirent ouvriers, ne cessèrent de se livrer au pénible labeur, et produisirent néanmoins, avec le secours de leurs lumières naturelles, augmentées par la lecture de très bons livres sans doute, des œuvres qui les honorent [...] on remarque Hans Schaz, de Nuremberg, né en 1494, mort en 1576. Il était cordonnier de son état [...] On vit briller en Angleterre, parmi les poètes populaires, le cultivateur Burn[s] [...] Olivier Basselin, maître d'un moulin à foulon, né à Vire, en Normandie, dans le XVe siècle [...] mérite une place distinguée parmi nos poètes. Vint ensuite Adam Billaut [...] Nous devons à maître André le perruquier le Tremblement de terre de Lisbonne [...] C'est à partir de 1830 surtout que les artisans poètes et prosateurs se sont manifestés en grand nombre. Il y aurait à faire une publication fort étendue et fort intéressante à leur sujet [...] en attendant ce vaste Panthéon des ouvriers, [...] revenons à notre menuisier, et donnons du dialogue que je lui ai consacré dès 1843 [...] la réimpression que voici. Puissent les ouvriers se distraire du travail des bras par celui de la pensée, conquérir l'art, la science, l'intelligence, s'élever, grandir comme je le souhaite du fond de mon âme, et bien se garder de dédaigner de se connaître eux-mêmes ", Maître Adam, Menuisier de Nevers. Dialogue, par Agricol PERDIGUIER, Menuisier, deuxième édition, Paris, Agricol Perdiguier, Libraire-Editeur, 1863, pp. 5-6.

33. George SAND, Histoire de ma vie, édition établie, présentée et annotée par Martine Reid, "Quarto", Paris, Éditions Gallimard, 2004, pp. 1260-1261. 
poésie» : l'obligation faite aux ouvriers-poètes, pour être identifiés comme d'authentiques poètes, de renoncer à l'exercice professionnel de la littérature qui était celui de leurs protecteurs parmi les auteurs établis, tirait précisément sa force sociale de sa capacité à être appropriée, mise en discours et en écriture par des acteurs qui en faisaient, ponctuellement ou non, un moyen d'améliorer leur sort.

L'événement "ouvriers-poètes» contribue ainsi à produire les discours et les pratiques constitutifs de la littérature contemporaine - au sens où le terme désigne une réalité sociale. Une idéologie du désintéressement sert en effet de commentaire permanent, dans les écrits qui font l'événement, à une constitution de la littérature en figure de la réussite sociale, et aussi en moyen d'action dans la société. Il ne s'agit pas là que d'invention textuelle : les mendiants de Sand montrent la large socialisation de cette figuration et de cette pratique de la littérature comme mode d'amélioration, même à une échelle qui peut paraitre dérisoire, de sa position sociale ${ }^{34}$. Plus largement, ce qui est ainsi socialisé, circulant des écrits publiés aux correspondances et des discours aux actions mises en œuvre pour bâtir une trajectoire ascendante, c'est l'écart entre activités intellectuelles et métier manuel. La poésie ouvrière situe l'investissement intellectuel de chacun, y compris des ouvriers, dans une zone à part, dans des pratiques distinguées de toutes les autres et traitées en pratiques quasi-aristocratiques : il y a là production sociale de la division manuel/intellectuel par des acteurs dont certains sont des ouvriers.

Il n'est peut-être pas sans intérêt de signaler pour finir qu'Adam Billaut n'était pas menuisier, mais entrepreneur domestique d'une famille princière devenu, grâce précisément à ses services poétiques, officier dans les institutions du duché-pairie détenu par cette famille, le duché de Nevers. Se forger une figure d'artisan-poète rimant sans avoir jamais rien appris, par pure inspiration, lui avait permis de réaliser cette trajectoire en donnant du prix à ces services poétiques. Cette figure, en effet, contribuait au discours faisant des belles-lettres une activité d'autant plus précieuse qu'elle dépendait du seul génie, non des titres ou du statut. Ce discours utile aux auteurs était produit par les auteurs : Billaut le menuisier, parce qu'il pouvait être employé dans ce cadre, s'était vu reconnaître - c'est-à-dire louer dans des poèmes publiés, notamment avec ses propres poèmes, manière d'en augmenter la valeur par le monde des lettres parisien, ce qui était un moyen de mieux servir ses patrons aristocrates. Ce qui est exceptionnel dans son cas, pour le XVII ${ }^{e}$ siècle, ce n'est donc pas le fait qu'un artisan ait écrit de la poésie : maints artisans, on l'a dit, en écrivaient et lui, en revanche, n'était pas artisan. Ce qui est exceptionnel ici, et à ce titre particulièrement éclairant sur les conditions

34. Voir Judith LYON-CAEN, La lecture et la vie. Les usages du roman au temps de Balzac, Paris, Éditions Tallandier, 2006. 
sociales de l'usage efficace de l'écriture au XVII ${ }^{e}$ siècle, c'est le succès de la mise en scène déterminée de cette figure. La rencontre entre l'événement de ce succès et l'événement "ouvriers-poètes" des années 1840 invite donc bien à dissocier fermement, c'est-à-dire à associer dans l'analyse, pratiques intellectuelles des artisans et ouvriers, dans leur diversité, et pratiques de mise en œuvre de ces pratiques, certaines dissimulées, d'autres publiées, dans des trajectoires sociales et politiques.

Dinah Ribard est maître de conférences à l'École des hautes études en sciences sociales et membre du GRIHL (Groupe de recherches interdisciplinaires sur l'histoire du littéraire). 